\title{
CORRECTION
}

\section{Correction to: Multicenter, Randomized, Controlled Study Comparing Tafluprost/Timolol Fixed Combination with Latanoprost/Timolol Fixed Combination in Primary Open-Angle Glaucoma and Ocular Hypertension}

Katsuyoshi Suzuki · Naomi Otsuka · Hiroko Hizaki · Masayo Hashimoto •

Yasuaki Kuwayama - On behalf of the Tafluprost/Timolol Versus Latanoprost/Timolol (TTVLT) Study Group

Published online: January 8, 2019

(c) Springer Healthcare Ltd., part of Springer Nature 2019

Correction to: Adv Ther (2018) 35:796-808

https://doi.org/10.1007/s12325-018-0718-9

In the original publication, the range to derive the $P$ values is incorrectly represented in Tables 2 and 3. The corrected tables are provided below.

The original article can be found online at https://doi. org/10.1007/s12325-018-0718-9.

K. Suzuki $(\bowtie)$

Suzuki Eye Clinic, Yamaguchi, Japan

e-mail: suzuki_eye@grace.ocn.ne.jp

N. Otsuka · H. Hizaki · M. Hashimoto

Santen Pharmaceutical Co., Ltd., Osaka, Japan

Y. Kuwayama

Fukushima Eye Clinic, Osaka, Japan 
Table 2 Mean ( \pm standard deviation) superficial punctate keratopathy scores (range 0-3)

\begin{tabular}{|c|c|c|c|c|c|}
\hline & TAF/TIM (n=58 & & $\mathrm{TT} / \mathrm{TIM}(\mathrm{n}=50)^{2}$ & & $p$-value ${ }^{b}$ \\
\hline \multicolumn{6}{|l|}{ Total SPK score } \\
\hline Week 0 & $0.7(0.9) 7$ & \multirow{3}{*}{$p=0.007^{c}$} & $0.8(1.1) 7$ & \multirow{3}{*}{$p=0.281^{c}$} & 0.661 \\
\hline Week 6 & $0.5(0.8) \quad p$ & & $0.8(1.1)$ & & - \\
\hline Week 12 & $0.4(0.7)$ & & $0.7(1.0)$ & & - \\
\hline Change from BL at Week 12 & $-0.2(0.7)$ & & $-0.2(1.1)$ & & 0.240 \\
\hline \multicolumn{6}{|l|}{ Superior SPK score } \\
\hline Week 0 & $0.1(0.2) \sqsupset$ & \multirow{3}{*}{$p=0.500^{c}$} & $0.1(0.2) \neg$ & \multirow{3}{*}{$p=1.000^{c}$} & 0.858 \\
\hline Week 6 & $0.0(0.1)$ & & $0.1(0.2)$ & & - \\
\hline Week 12 & $0.0(0.1)$ & & $0.1(0.2)$ & & - \\
\hline Change from BL at Week 12 & $0.0(0.2)$ & & $0.0(0.3)$ & & 0.461 \\
\hline \multicolumn{6}{|l|}{ Mid SPK score } \\
\hline Week 0 & $0.2(0.5)-7$ & \multirow{3}{*}{$p=0.549^{c}$} & $0.4(0.6)-7$ & \multirow{3}{*}{$p=0.124^{c}$} & 0.040 \\
\hline Week 6 & $0.2(0.4)$ & & $0.3(0.6)$ & & - \\
\hline Week 12 & $0.2(0.4)$ & & $0.2(0.4)$ & & - \\
\hline Change from BL at Week 12 & $-0.1(0.4)$ & & $-0.2(0.7)$ & & 0.416 \\
\hline \multicolumn{6}{|l|}{ Inferior SPK score } \\
\hline Week 0 & $0.4(0.6) \sqsupset$ & \multirow{3}{*}{$p=0.035^{c}$} & $0.4(0.6)-7$ & \multirow{3}{*}{$p=1.000^{c}$} & 0.879 \\
\hline Week 6 & $0.3(0.6)$ & & $0.4(0.6)$ & & - \\
\hline Week 12 & $0.3(0.5)$ & & $0.4(0.6)$ & & - \\
\hline Change from BL at Week 12 & $-0.2(0.5)$ & & $0.0(0.6)$ & & 0.085 \\
\hline
\end{tabular}

Bold indicates significant values

$B L$ baseline, $L A T$ latanoprost, SPK superficial punctate keratopathy, TAF tafluprost, TIM timolol

${ }^{a}$ Data missing for one patient in the LAT/TIM group

${ }^{b}$ Calculated using Wilcoxon two-sample test

c Calculated using Wilcoxon one-sample test 
Table 3 Mean ( \pm standard deviation) tear break-up times and hyperemia scores (range 0-3)

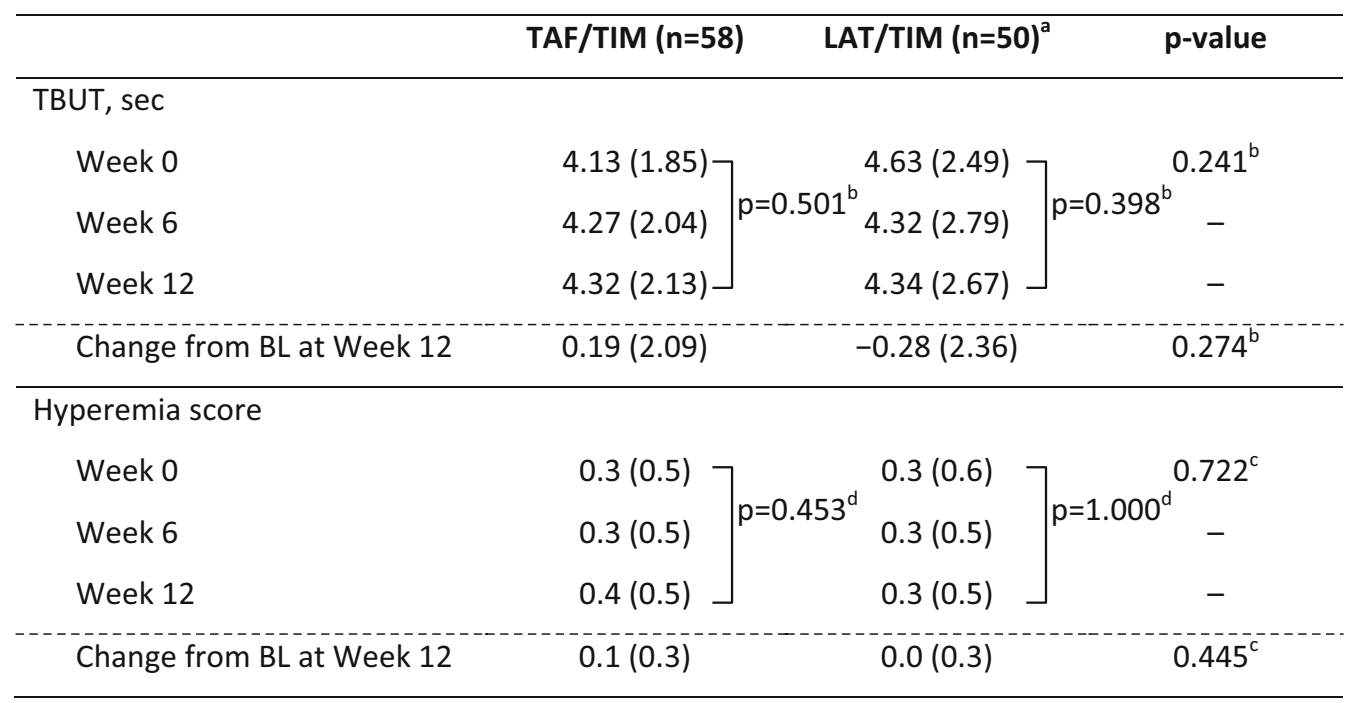

$B L$ baseline, $L A T$ latanoprost, TAF tafluprost, TBUT tear breakup time, TIM timolol

${ }^{a}$ Data missing for one patient in the LAT/TIM group

${ }^{\mathrm{b}}$ Calculated using $t$ test

${ }^{c}$ Calculated using Wilcoxon two-sample test

${ }^{d}$ Calculated using Wilcoxon one-sample test 